\title{
Role of surface passivation in the formation of Dirac states at polar surfaces of topological crystalline insulators: The case of $\operatorname{SnTe}(111)$
}

\author{
S. V. Eremeev, ${ }^{1,2}$ Yu. M. Koroteev, ${ }^{1,2}$ I. A. Nechaev, ${ }^{2,3}$ and E. V. Chulkov ${ }^{2,3,4}$ \\ ${ }^{1}$ Institute of Strength Physics and Materials Science, 634021, Tomsk, Russia \\ ${ }^{2}$ Tomsk State University, Laboratory for Nanostructured Surfaces and Coatings, 634050 Tomsk, Russia \\ ${ }^{3}$ Donostia International Physics Center, 20018 San Sebastián/Donostia, Basque Country, Spain \\ ${ }^{4}$ Departamento de Física de Materiales UPV/EHU, Centro de Física de Materiales CFM-MPC, and Centro Mixto CSIC-UPV/EHU, \\ 20080 San Sebastián/Donostia, Basque Country, Spain
}

(Received 4 February 2014; revised manuscript received 14 April 2014; published 28 April 2014)

\begin{abstract}
We present $a b$ initio density functional theory (DFT) calculation results for electronic and spin structures of both the Te- and Sn-terminated SnTe(111) polar surfaces. Rocksalt narrow-gap semiconductor SnTe belongs to the recently discovered class of topological crystalline insulators in which the topological nature of surface electronic states arises from the crystal symmetry combined with band inversion at the $L$ point. We demonstrate that in contrast to earlier model calculations only trivial spin-split states propagating over the entire two-dimensional Brillouin zone emerge at the $\operatorname{SnTe}(111)$ surfaces owing to the surface potential effect which destroys weakly protected topological states. We show that the surface passivation eradicates the trivial surface states and recovers the even number of the helical spin-polarized topological Dirac cones centered at the $\bar{\Gamma}$ and $\bar{M}$ points prescribed for the topological crystalline insulator by the crystal symmetry.
\end{abstract}

DOI: 10.1103/PhysRevB.89.165424

PACS number(s): 71.20.Nr, 73.20.At, 85.75.-d

\section{INTRODUCTION}

The tin chalcogenides (SnTe, $\mathrm{SnSe}, \mathrm{SnS}$ ) are small band gap semiconductors which have long been of great interest due to their fascinating thermodynamic, vibration, electronic, and infrared properties [1,2]. These materials are important in several practical applications. In particular, SnTe and its alloys are well known for their use as infrared detectors, infrared lasers, and microelectronic and thermoelectric devices [3,4].

The discovery of $\mathbb{Z}_{2}$ topological insulators (TI) has prompted the search for topological phases with surface states protected by non-time-reversal symmetries. Recently. it was shown that in some materials the crystal mirror symmetry can create topologically protected surface states [5]. These materials, SnTe [6] and $\mathrm{Pb}_{1-x} \mathrm{Sn}_{x} \mathrm{Te}(\mathrm{Se})[7,8]$, called topological crystalline insulators (TCI), have recently been expanded to include other tin chalcogenides, $\mathrm{SnS}$ and SnSe [9]. The nontrivial electronic band structure of TCI is characterized by an integer topological invariant known as the mirror Chern number [10], which arises from band inversion at the $L$ point of the bulk Brillouin zone (BZ) combined with the mirror symmetry in the rocksalt fcc crystal structure $(F m \overline{3} m)$ with respect to the (110) plane. Owing to the band structure topology, the topologically protected surface states should arise on the (001), (111), and (110) surfaces, which preserve the mirror symmetry.

In particular, on the (001) surface four Dirac cones should arise at so-called $\bar{\Lambda}$ points, which are close to the $\overline{\mathrm{X}}$ points in the $\bar{\Gamma}-\overline{\mathrm{X}}$ directions. The (001) surface states, predicted by $\mathbf{k} \cdot \mathbf{p}$ theory and tight-binding model calculations, were observed in angle-resolved photoemission spectroscopy (ARPES) measurements on SnTe [11], $\mathrm{Pb}_{1-x} \mathrm{Sn}_{x} \mathrm{Te}$ [12], and $\mathrm{Pb}_{1-x} \mathrm{Sn}_{x} \mathrm{Se}$ [13]. The helical spin texture in these Dirac cones was also observed experimentally [12].

The (001) and (110) surfaces comprise an equal number of $\mathrm{Sn}$ and Te atoms on each surface. In contrast, the (111) surface can be viewed as a sequence of equidistant anion
$\left(\mathrm{Te}^{-}\right)$and cation $\left(\mathrm{Sn}^{+}\right)$hcp layers alternating along the space diagonal to the cubic cell, and thus the surface of SnTe is formed either by the $\mathrm{Sn}^{+}$or $\mathrm{Te}^{-}$atomic layer. The (111) surface has been predicted to support Dirac cones centered at the $\bar{\Gamma}$ and $\bar{M}$ points of two-dimensional (2D) BZs [7,8,14]. However, this prediction is based on the model calculations without taking into account self-consistent charge density and structural optimization; that is, the surface chemistry has not been included.

Very recently, the $\bar{\Gamma}$ and $\bar{M}$ Dirac states at SnTe[15] and $\mathrm{Pb}_{1-x} \mathrm{Sn}_{x}$ Se [16] surfaces with orientation (111) were observed experimentally in ARPES measurements. However, the surface termination and structure details have not been unambiguously characterized in these studies.

In this paper, we present detailed $a b$ initio DFT calculation results for the clean Te- and Sn-terminated (111) surfaces of the SnTe crystalline insulator as well as for these surfaces covered with hydrogen and fluorine monolayers. We show that the ideal polar Te and $\mathrm{Sn}$ surfaces produce strong dangling-bond surface states propagating in the energy gap over the entire 2D Brillouin zone. Relaxation of these surfaces, optimization of the surface and near-surface atomic layer positions, does not remove the dangling-bond surface states; however, it results in a conelike state at the $\bar{\Gamma}$ point. Further we demonstrate that passivation of these surfaces with $\mathrm{H}$ or $\mathrm{F}$ eradicates the trivial surface states and leads to the emergence of the helical spin-polarized Dirac-like surface states at the $\bar{\Gamma}$ and $\bar{M}$ points. We also discuss recent experiments on the TCI (111) surface. We argue that the absence of the dangling-bond surface states in the ARPES spectra can be caused by a complicated mixed cation/anion termination structure of the surface that makes it nonpolar.

\section{METHODS}

For structural optimization and electronic band calculations we use the VIENNA AB INITIO SIMULATION PACKAGE (VASP) 
[17] with generalized gradient approximation (GGA) to the exchange-correlation potential. The interaction between the ion cores and valence electrons was described by the projector augmented-wave method $[18,19]$. The experimental lattice parameter of the $\mathrm{NaCl}$ cubic phase of SnTe $(6.313 \AA)$ was used for calculation [20]. Relativistic effects, including spinorbit coupling, were taken into account. To simulate polar $\mathrm{SnTe}(111)$ surfaces we consider 47 atomic layer symmetric slabs, so that the upper and lower surfaces are identical (Te or Sn terminated). A vacuum spacer of $\sim 20 \AA$ was included to ensure negligible interaction between slabs. The $k$-point meshes of $7 \times 7 \times 7$ and $9 \times 9 \times 1$ were used for the bulk and slab calculations, respectively. The total-energy convergence was better than $1.0 \times 10^{-6} \mathrm{eV}$. The atomic positions for the first five surface layers were obtained during a relaxation procedure until forces became less than $1.0 \times 10^{-5} \mathrm{eV} / \AA$. Complementary calculations of both the bulk and surface SnTe(111) electronic structures were performed using the fullpotential linearized augmented-plane-wave (FLAPW) method as implemented in the FLEUR code [21]. To additionally examine the validity of our DFT calculations, we also calculated the surface electronic structure of a nonpolar surface $\mathrm{SnTe}(001)$.

In order to estimate the accuracy of our DFT quantitative description of the bulk band gap of SnTe, we performed $a b$ initio calculations of many-body corrections to the GGA band structure within a scheme which we have already applied to binary and ternary topological insulators [22-24]. The manybody corrections were found within the one-shot $G W$ approach as realized by the SPEX code [25]. The spin-orbit interaction was included into the $G W$ calculations already at the DFT level [26]. The GGA calculations were carried out using the FLEUR code with a plane-wave cutoff of $k_{\max }=3.7 \mathrm{bohr}^{-1}$, an angular momentum cutoff of $l_{\max }=14$, equal muffin-tin radii of $2.9 \AA$ for $\mathrm{Sn}$ and Te, and a $7 \times 7 \times 7 \mathbf{k}$-point mesh. The FLAPW basis was extended by the conventional local orbitals $[27,28]$ to treat the core $4 s, 4 p$, and $4 d$ states. The energy cutoff between core and valence states was put at $-6.4 \mathrm{Ha}$, which corresponds to 46 valence electrons in the considered energy window. In order to accurately describe high-lying unoccupied states [29], two local orbitals per angular momentum up to $l=3$ were included for each atom. The Fermi level was placed in the middle of the band gap. In the $G W$ calculations, the dielectric matrix was evaluated within the random-phase approximation and represented with the use of the mixed product basis $[25,30]$, where we chose an angular momentum cutoff in the muffin-tin spheres of 4 and a linear momentum cutoff of $3.5 \mathrm{bohr}^{-1}$. The $G W$ quasiparticle spectrum presented in this paper was obtained with number of bands $N_{b}=700$ (occupied and unoccupied bands within the energy window of $350 \mathrm{eV}$ centered at the Fermi level) and a less dense k-point mesh $(5 \times 5 \times 5)$ than in the GGA calculations. The analysis of the band gap convergence with respect to $N_{b}$ has shown that the band gap decreases with increasing $N_{b}$, and the parameters listed above ensure the band gap converged within $5 \mathrm{meV}$.

\section{RESULTS AND DISCUSSION}

The GGA bulk band structure of SnTe calculated by both the VASP and FLEUR codes along high-symmetry directions of the three-dimensional (3D) Brillouin zone [Fig. 1(a)] is
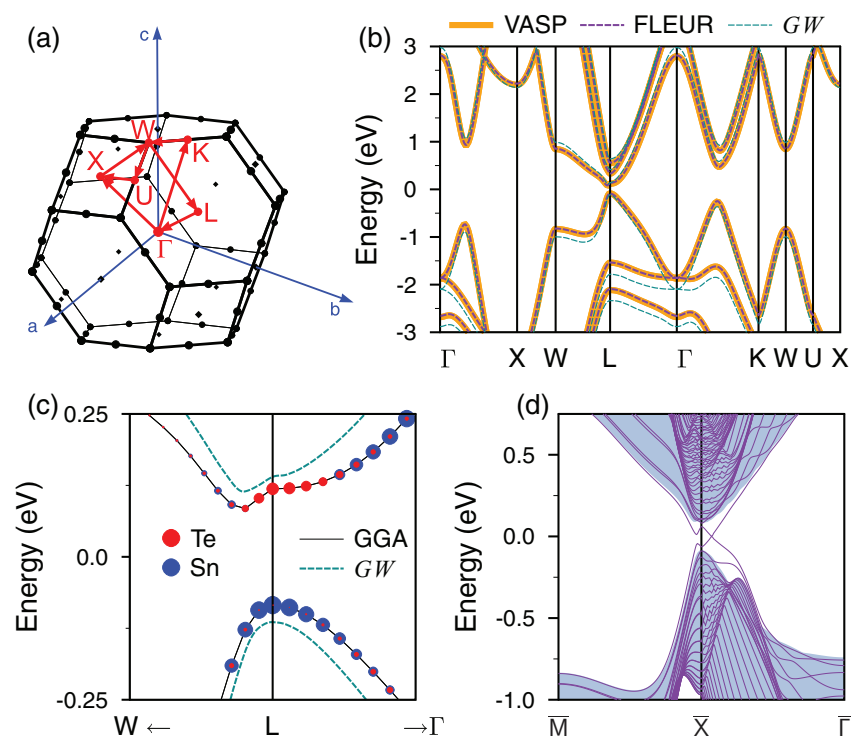

FIG. 1. (Color online) (a) Bulk Brillouin zone of rocksalt SnTe. (b) GGA electronic bulk band structure calculated along the highsymmetry directions marked in panel (a) using VASP and FLEUR (orange solid and violet dashed lines, respectively). The $G W$ quasiparticle spectrum is presented by dark cyan dashed lines. (c) Band gap inversion at the $L$ point. For a detailed comparison of the GGA band gap with the $G W$ one, the quasiparticle bands are also shown. (d) Band structure of the nonpolar $\mathrm{SnTe}(001)$ surface.

presented in Fig. 1(b). As is seen in Fig. 1(b), the results of the VASP and FLEUR calculations are in excellent agreement with each other. The spectrum demonstrates an almost direct spinorbit-derived narrow gap (170 meV from VASP and $176 \mathrm{meV}$ from FLEUR) that lies in the vicinity of the $L$ point of the Brillouin zone. As seen in Fig. 1(b), the $G W$ corrections give rise to a nearly uniform moderate shift of the valence and the conduction bands with respect to each other, except in the vicinity of the $L$ point, where the shift is relatively small. It results in a slight increase of the band gap up to $229 \mathrm{meV}$ [see Fig. 1(c)]. The experimental band gap in SnTe is strongly dependent on the hole carrier concentration and varies in the range of $0.10-0.30 \pm 0.05 \mathrm{eV}$ [31-36]. Both theoretical values of the bulk gap, obtained within the GGA and $G W$ approaches, lie in the middle of the experimental range.

The gap at the $L$ point, which is primarily composed of the $p$ orbitals, is inverted. While throughout the Brillouin zone $\mathrm{Te}(\mathrm{Sn})$ orbitals are occupied (unoccupied) in the vicinity of $L$, the valence band edge is composed of Sn orbitals, and the conduction band edge is derived from Te orbitals [Fig. 1(c)].

In contrast to the time-reversal topological insulators the band inversion in SnTe occurs at an even number of timereversal-invariant momenta (TRIMs) and leads to a trivial $\mathbb{Z}_{2}$ topological invariant [6]. At nonpolar surfaces the bulk band inversion results in the formation of an even number (as distinct from time-reversal TIs having an odd number of Dirac states) of the Dirac-like gapless surface states [6]. For instance, at the (001) surface these states arise at the $\overline{\mathrm{X}}-\bar{\Gamma}$ lines, at a distance $k_{\|} \approx 0.05 \AA^{-1}$ from the $\overline{\mathrm{X}}$ point, as shown in Fig. 1(d). This value as well as the entire surface spectrum of $\operatorname{SnTe}(001)$ is in excellent agreement with recent studies $[6,8,37]$. 

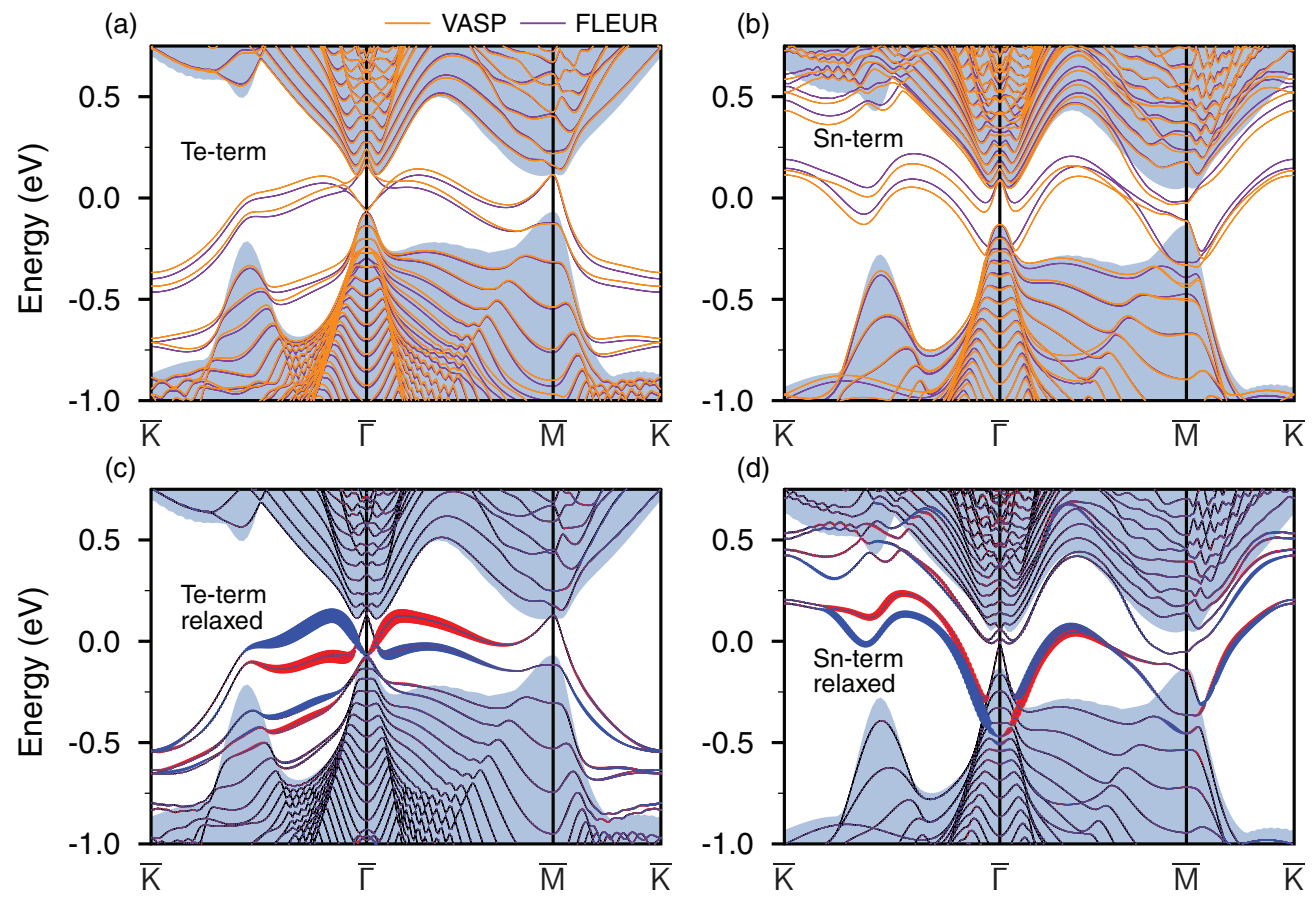

FIG. 2. (Color online) Electronic structure of Te- and Sn-terminated polar SnTe(111) surfaces: ideal (a) Te- and (b) Sn-terminated surfaces calculated using VASP and FLEUR (steel blue area is the projection of the bulk states) and relaxed (c) Te- and (d) Sn-terminated surfaces. Circles represent the weights of the states within the four outermost surface atomic layers multiplied by the value of the in-plane spin component (red and blue colors denote positive and negative values of spin, respectively).

According to the $\mathbf{k} \cdot \mathbf{p}$ theory prediction and tight-binding calculations [8] at the (111) surface, in contrast to the (001) and (110) surfaces, the topologically protected Dirac cones appear at the $\bar{\Gamma}$ and at three $\bar{M}$ points, which are the appropriate projection points of the corresponding $L$ points belonging to the three $\{110\}$ mirror planes of the (111) surface. For the Te-terminated $\operatorname{SnTe}(111)$ four topological surface states with the Dirac points (DPs) lying just below the conduction band are obtained in the tight-binding calculations, while at the Sn-terminated surface the Dirac points appear close to the top of the valence band [8]. Similar results were obtained for anion- and cation-terminated (111) surfaces in $\mathrm{Pb}_{0.4} \mathrm{Sn}_{0.6}$ Te [7]. It should be stressed that in these calculations based on the nearest-neighbor tight-binding approach developed for bulk materials, the surface self-consistent potential effect for the polar surfaces was not taken into account.

The crystal structure of polar NaCl-type semiconductor compounds can be described as an alternating sequence of anion and cation (111) layers (in particular, for SnTe, our calculation performed within the Bader scheme [38] shows $\mathrm{Te}^{-0.74}$ and $\mathrm{Sn}^{+0.74}$ ). A direct consequence of this geometry is that nonreconstructed polar surfaces should be electrostatically unstable [39]. Indeed, numerous experimental studies show that clean polar surfaces are reconstructed. There are, however, a few exceptions which do not follow this rule. Such surfaces include the $\operatorname{SnTe}(111)$ surfaces which do not undergo any reconstruction [40].

The typical effect resulting from the lower coordination of the surface layer is surface relaxation, which can affect the surface electronic structure. At both terminations the surface relaxation we obtain has alternating-sign character, starting with a contraction of the first interlayer spacing. The relaxation displacements are $\sim \mp 0.2(0.3) \AA$ in near-surface layers at the Sn-terminated (Te-terminated) surface and decay gradually upon moving toward the bulk. It is noteworthy that in this case the near-surface structure of the polar $\mathrm{SnTe}(111)$ surfaces qualitatively resembles the structure of rhombohedral $\alpha$-GeTe(111) [41], where the Ge and Te ions are displaced from the ideal rocksalt sites along the [111] direction, forming bilayers with an alternating stacking sequence of cation and anion layers with "short" and "long" interlayer distances.

The calculated electronic band spectra of the Te- and Sn-terminated surfaces of $\operatorname{SnTe}(111)$ are presented in Fig. 2. Figures 2(a) and 2(b) show the electronic structure of the unrelaxed slabs as calculated both by VASP and FLEUR, while Figs. 2(c) and 2(d) represent the VASP-derived spectra for both terminations with surface relaxation included. Comparing our results which take into account the effect of self-consistent charge-density distribution with earlier tight-binding calculations, we find that the electronic spectra are completely different. Instead of predicted isolated Dirac cones at the $\bar{\Gamma}$ and $\bar{M}$ points, both Te- and Sn-terminated surfaces hold the spin-split surface states, which propagate over the projected bulk energy gap around the Fermi level. The VASP and FLEUR methods give similar dispersions for these states which are slightly influenced by the surface relaxation. The only noticeable result of the surface relaxation for both terminations is the emergence of a state with linear dispersion within the band gap in close vicinity to the $\bar{\Gamma}$ point, which can be considered a "precursor" state for the formation of the $\bar{\Gamma}$ Dirac cone. 
(a)

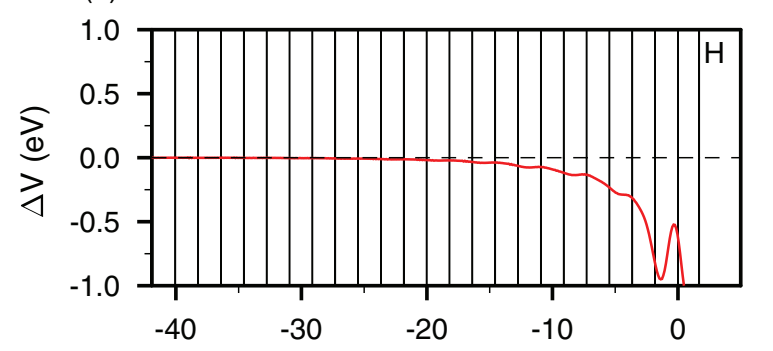

(c)

z (A)

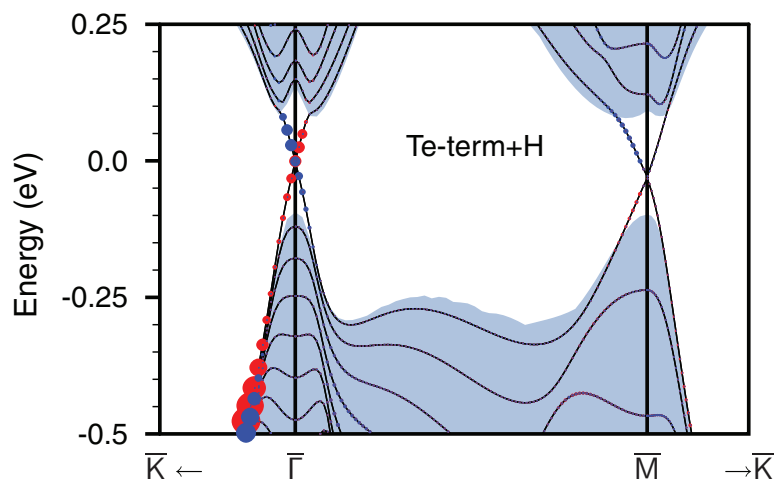

(e)

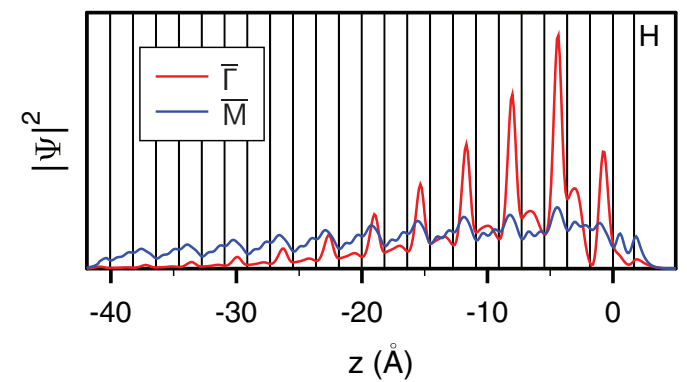

(b)

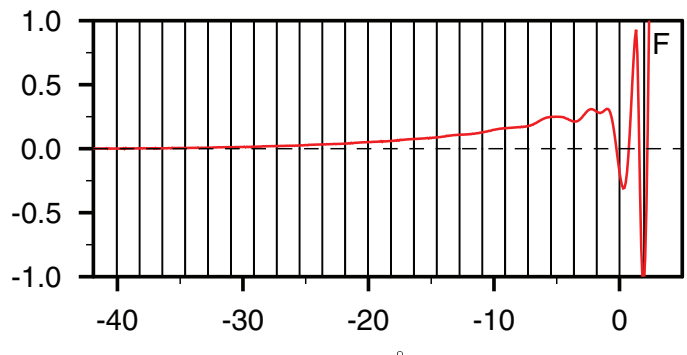

z $(\AA)$

(d)

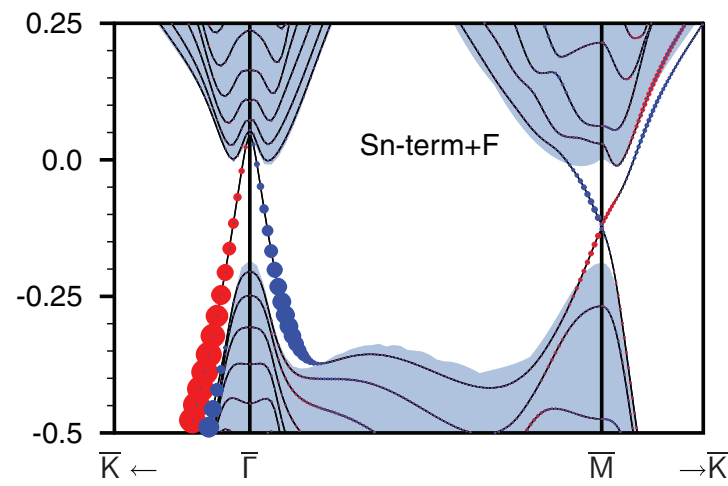

(f)



FIG. 3. (Color online) Near-surface potential changes caused by passivation of (a) Te- and (b) Sn-terminated surfaces. Electronic structure of (c) Te- and (d) Sn-terminated surfaces, passivated by hydrogen and fluorine, respectively. Spatial localization of the topological $\bar{\Gamma}$ and $\bar{M}$ states near the band crossing for passivated (e) Te- and (f) Sn-terminated surfaces. In (a) and (b) and (e) and (f) vertical lines mark the positions of the atomic planes; $z=0$ corresponds to the outermost $\mathrm{Te}(\mathrm{Sn})$ layer.

Similar dangling-bond spin-split surface states with dispersion dependent on the surface termination were obtained within DFT calculations at the surfaces of $\mathbb{Z}_{2}$ TIs of the Tl-based family [42-44]. However, in the latter case these states coexist with the $\bar{\Gamma}$ Dirac cone, protected by time-reversal symmetry. In contrast, at the SnTe(111) polar surfaces the surface self-consistent potential effect destroys the topological states weakly protected by the crystal symmetry.

It is worth noting that in Tl-based $\mathbb{Z}_{2}$ TIs the ARPES measurements [45-47] did not reveal the existence of surface states in the fundamental energy gap except for the topologically protected Dirac cone. A detailed core-level photoelectron spectroscopy and scanning tunneling microscopy study [48] on the $\mathrm{TlBiSe}_{2}$ surface has shown that the discrepancy between the calculation results and experiments is caused by complicated surface morphology. It was found that the cleaved surface is the Se-terminated surface covered with $\mathrm{Tl}$ atoms forming islands so that the surface has an $~ 50: 50 \mathrm{Se}-\mathrm{Tl}$ termination. We can assume that the discrepancy between our $a b$ initio results for polar $\operatorname{SnTe}(111)$ surfaces and recent ARPES data [15], which demonstrate only the $\bar{\Gamma}$ and $\bar{M}$ Dirac cones in spectra, can be attributed to the mixed Sn-Te surface termination in the studied samples. Such a complicated surface structure results in the presence of equal (or almost equal) numbers of $\mathrm{Sn}$ and Te atoms, which makes the surface nonpolar, like the (001) and (110) surfaces. Therefore further experimental study of surface morphology of the TCI (111) surfaces and its possible influence on topological electronic and transport properties is necessary.

Next, we have studied modification of the surface electronic structure and surface potential of the polar surfaces caused by passivation of the dangling bonds of the surface-layer atoms. The passivation of the Te-terminated surface by hydrogen results in modification of the near-surface potential $\Delta V=$ $V_{\text {pass }}-V_{\text {pr }}$ shown in Fig. 3(a) (where $V_{\text {pass }}$ and $V_{\text {pr }}$ are electrostatic potentials of passivated and pristine surfaces, respectively). It is worth noting that the passivation results in reducing the relaxation of the surface atomic layers, bringing 

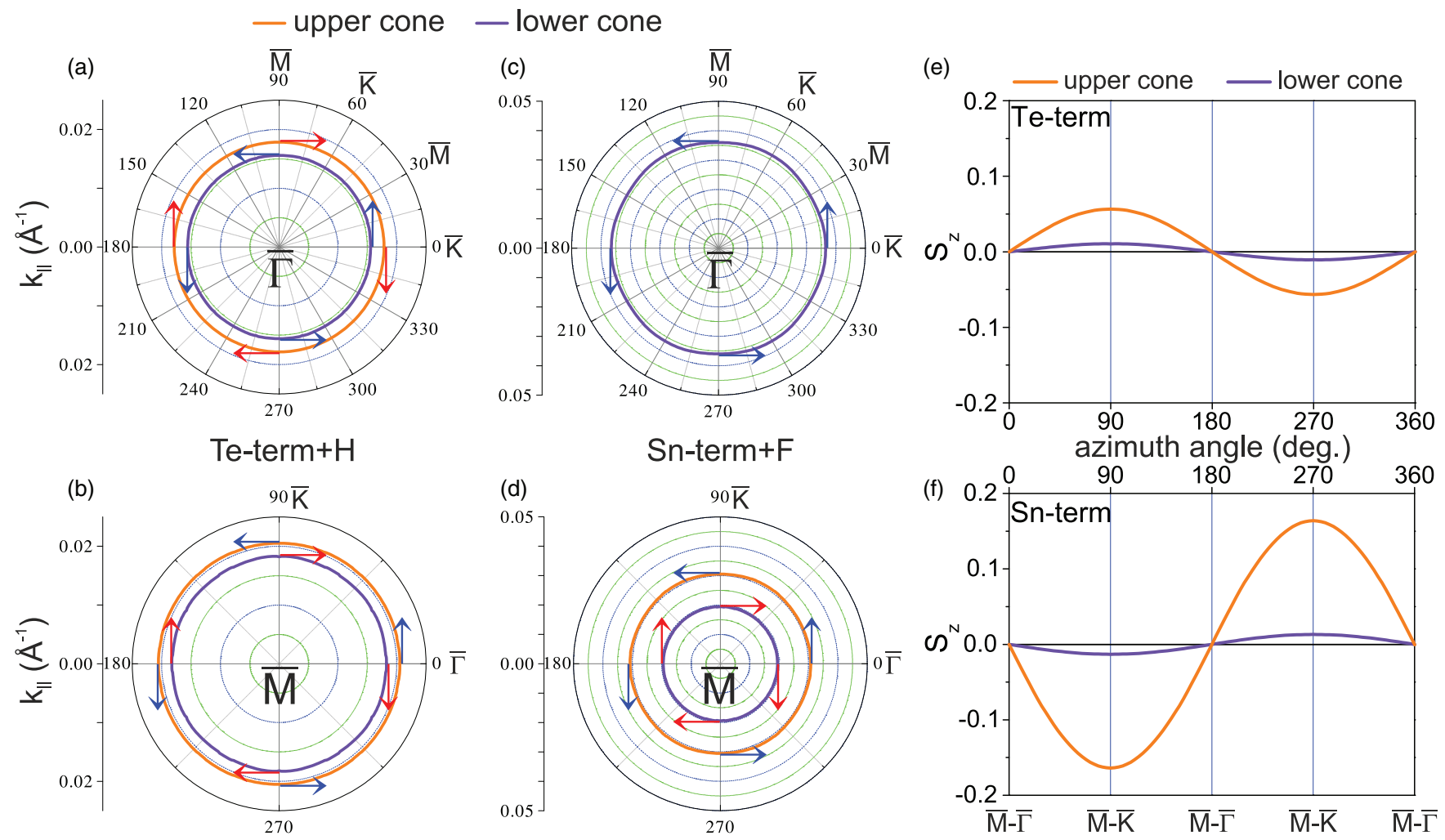

FIG. 4. (Color online) Constant-energy contours (CECs) for the (a) and (c) $\bar{\Gamma}$ and (b) and (d) $\bar{M}$ Dirac cones at passivated (a) and (b) Te- and (c) and (d) Sn-terminated surfaces. CECs are taken at $\pm 40 \mathrm{meV}$ with respect to the Dirac point (except for the $\bar{\Gamma}$-cone case at the Sn-terminated surface, where the CEC is given for the middle of the bulk gap). Red and blue arrows show in-plane clockwise and counterclockwise spin polarizations, respectively. Azimuthal dependence of the out-of-plane spin component $S_{z}$ for the $\bar{M}$ Dirac cone at passivated (e) Te- and (f) Sn-terminated surfaces.

the interlayer distances closer to their bulk value. Thus the $\mathrm{Te}_{1}-\mathrm{Sn}_{2}$ and $\mathrm{Sn}_{2}-\mathrm{Te}_{3}$ interlayer spacing relaxations are -0.07 and $+0.06 \AA$, respectively, and they are only $\sim 0.001 \AA$ in deeper layers. Under these conditions the trivial spin-split surface states disappear from the bulk energy gap, and welldeveloped spin-polarized Dirac cones arise at the $\bar{\Gamma}$ and $\bar{M}$ points [Fig. 3(c)]. The Dirac points of the topological states lie in the middle of the band gap: the $\bar{\Gamma} \mathrm{DP}$ is situated at the Fermi level, whereas the $\bar{M}$ DP lies just $30 \mathrm{meV}$ below $E_{\mathrm{F}}$.

Hydrogenation of the Sn-terminated slab leads to the emergence of the $\bar{\Gamma}$ topological state with the Dirac point sunken into the bottom of the conduction band, while trivial surface states still exist within the band gap and the $\bar{M}$ cone does not appear (not shown). To remove the trivial spin-split states from the band gap we substitute the hydrogen passivation monolayer for the monolayer of the more electronegative fluorine, which causes $\Delta V$ to have an opposite sign [Fig. 3(b)] and results in a sizable reduction of the surface interlayer relaxation. In this case the $\mathrm{Sn}$-terminated surface becomes free of band gap trivial surface states, and topological states arise at the $\bar{\Gamma}$ and $\bar{M}$ points [Fig. 3(d)]. In this system the Fermi level lies just below the bottom of the conduction band. The $\bar{M}$ topological state DP is situated in the middle of the band gap, like in the passivated Te-terminated case, whereas the $\bar{\Gamma}$ DP snuggles up to the bottom of the conduction band, so that only the lower part of the Dirac cone spreads across the band gap.
The topological states at the passivated Te- and Snterminated surfaces penetrate deeply into the bulk up to $\approx 40 \AA$ [Figs. 3(e) and 3(f)]. Such a localization of the topological states is distinct from the case of quintuple(septuple)-layered [Q(S)L] TIs of $\mathrm{Bi}_{2} \mathrm{Se}_{3}$ type, where the Dirac state is mainly located within the outermost $\mathrm{Q}(\mathrm{S}) \mathrm{L}[49,50]$ and is similar to the deeply penetrated Dirac states in $\mathrm{TlBi}(\mathrm{Sb}) \mathrm{Te}(\mathrm{Se})_{2}$ TIs [42].

Finally, we examine the electron-spin texture for the topological states at the passivated $\operatorname{SnTe}(111)$ surfaces. In Figs. 4(a)-4(d) we show spin-resolved constant-energy cuts (CECs) through the Dirac cones. The CECs demonstrate a circular shape for the $\bar{\Gamma}$ and $\bar{M}$ cones. Both the $\bar{\Gamma}$ and $\bar{M}$ spin-polarized states have a helical spin texture. One can see the $\bar{\Gamma}$ states demonstrate positive (clockwise) spin helicity above the DP and negative helicity in the lower part of the cone. In the $\bar{M}$ cone all the helicities are reversed compared to the case of the $\bar{\Gamma}$ Dirac cone.

For the (111) surface the symmetry requires that the out-ofplane spin component $S_{z}$ should be equal to zero along the three high-symmetry $\bar{\Gamma}-\bar{M}$ directions and $S_{z}$ should be zero for the $\bar{\Gamma}$ cone, while nonzero $S_{z}$ can appear in the Dirac cone around the $\overline{\mathrm{M}}$ point beyond the $\bar{\Gamma}-\overline{\mathrm{M}}$ line [6]. Indeed, the azimuthal scan of the calculated $z$ components of the spin expectation values for the $\bar{M}$ cone shows sinusoidal behavior with zeros in the $\bar{M}-\bar{\Gamma}$ and maxima (minima) in the $\overline{\mathrm{M}}-\overline{\mathrm{K}}$ directions [Figs. 4(e) and 4(f)]. At both Te- and Sn-terminated surfaces the amplitude of the lower cone azimuthal dependencies is considerably smaller 
than that of the upper cone. In turn, the deviation of the spin direction from the in-plane alignment is largest in the Snterminated case. This fact together with the observed antiphase modulation of $S_{z}$ on differently terminated surfaces is related to peculiarities of the variation of the in-plane potential which gets rise to the out-of-plane spin component.

\section{SUMMARY}

In conclusion, on the basis of ab initio DFT calculations we have studied the electronic and spin structures of the $\operatorname{SnTe}(111)$ polar surfaces. We have shown that at pristine surfaces the surface potential effect destroys the topological states weakly protected by the crystal symmetry and only trivial spin-split states emerge within the bulk energy gap. We have found that the four helical spin-polarized topological Dirac states can arise on these surfaces only when the surface is specifically passivated. We expect that (1) the revealed peculiarities of electronic structure of the pristine and passivated polar surfaces of SnTe will be common for the tin chalcogenide class of TCI materials and (2) the use of different passivation elements can open new ways to manipulate the topological surface-state characteristics on polar surfaces of TCI. We believe that our findings will stimulate further theoretical and experimental investigations of polar TCI surfaces as promising systems for potential spintronic and optoelectronic applications.

\section{ACKNOWLEDGMENTS}

We acknowledge partial support from the Basque Country government, Departamento de Educación, Universidades e Investigación (Grant No. IT-366-07), the Spanish Ministerio de Ciencia e Innovación (Grant No. FIS2010-19609-C02-00), the Ministry of Education and Science of the Russian Federation (Grant No. 2.8575.2013), and the Russian Foundation for Basic Research (Grant No. 13-02-12110-ofi-m).
[1] P. K. Nair, M. Ocampo, A. Fernandez, and M. T. S. Nair, Sol. Energy Mater. 20, 235 (1990).

[2] L. D. Hicks, T. C. Harman, X. Sun, and M. S. Dresselhaus, Phys. Rev. B 53, R10493 (1996).

[3] T. K. Chaudhuri, Int. J. Energy Res. 16, 481 (1992).

[4] G. P. Agrawal and N. K. Dutta, Semiconductor Lasers (Van Nostrand Reinhold, New York, 1993).

[5] L. Fu, Phys. Rev. Lett. 106, 106802 (2011).

[6] T. H. Hsieh, H. Lin, J. Liu, W. Duan, A. Bansil, and L. Fu, Nat. Commun. 3, 982 (2012).

[7] S. Safaei, P. Kacman, and R. Buczko, Phys. Rev. B 88, 045305 (2013).

[8] J. Liu, W. Duan, and L. Fu, Phys. Rev. B 88, 241303 (2013).

[9] Y. Sun, Z. Zhong, T. Shirakawa, C. Franchini, D. Li, Y. Li, S. Yunoki, and X.-Q. Chen, Phys. Rev. B 88, 235122 (2013).

[10] J. C. Y. Teo, L. Fu, and C. L. Kane, Phys. Rev. B 78, 045426 (2008).

[11] Y. Tanaka, Zhi Ren, T. Sato, K. Nakayama, S. Souma, T. Takahashi, K. Segawa, and Y. Ando, Nat. Phys. 8, 800 (2012).

[12] S. Y. Xu, C. Liu, N. Alidoust, M. Neupane, D. Qian, I. Belopolski, J. D. Denlinger, Y. J. Wang, H. Lin, L. A. Wray, G. Landolt, B. Slomski, J. H. Dil, A. Marcinkova, E. Morosan, Q. Gibson, R. Sankar, F. C. Chou, R. J. Cava, A. Bansil, and M. Z. Hasan, Nat. Commun. 3, 1192 (2012).

[13] P. Dziawa, B. J. Kowalski, K. Dybko, R. Buczko, A. Szczerbakow, M. Szot, E. Łusakowska, T. Balasubramanian, B. M. Wojek, M. H. Berntsen, O. Tjernberg, and T. Story, Nat. Mater. 11, 1023 (2012).

[14] A. A. Taskin, S. Sasaki, K. Segawa, and Y. Ando, Phys. Rev. B 89, 121302(R) (2014).

[15] Y. Tanaka, T. Shoman, K. Nakayama, S. Souma, T. Sato, T. Takahashi, M. Novak, K. Segawa, and Y. Ando, Phys. Rev. B 88, 235126 (2013).

[16] C. M. Polley, P. Dziawa, A. Reszka, A. Szczerbakow, R. Minikayev, J. Z. Domagala, S. Safaei, P. Kacman, R. Buczko, J. Adell, M. H. Berntsen, B. M. Wojek, O. Tjernberg, B. J. Kowalski, T. Story, and T. Balasubramanian, Phys. Rev. B 89, 075317 (2014).
[17] G. Kresse and J. Hafner, Phys. Rev. B 48, 13115 (1993).

[18] P. E. Blöchl, Phys. Rev. B 50, 17953 (1994).

[19] G. Kresse and D. Joubert, Phys. Rev. B 59, 1758 (1999).

[20] R. W. G. Wyckoff, Crystal Structures, 2nd ed. (Interscience, New York, 1963), Vol. 1, pp. 85-237.

[21] http://www.flapw.de

[22] I. A. Nechaev, R. C. Hatch, M. Bianchi, D. Guan, C. Friedrich, I. Aguilera, J. L. Mi, B. B. Iversen, S. Blügel, Ph. Hofmann, and E. V. Chulkov, Phys. Rev. B 87, 121111(R) (2013).

[23] I. P. Rusinov, I. A. Nechaev, and E. V. Chulkov, JETP Lett. 98, 397 (2013).

[24] I. A. Nechaev and E. V. Chulkov, Phys. Rev. B 88, 165135 (2013).

[25] C. Friedrich, S. Blügel, and A. Schindlmayr, Phys. Rev. B 81, 125102 (2010).

[26] R. Sakuma, C. Friedrich, T. Miyake, S. Blügel, and F. Aryasetiawan, Phys. Rev. B 84, 085144 (2011).

[27] D. Singh, Phys. Rev. B 43, 6388 (1991).

[28] E. Sjöstedt, L. Nordström, and D. J. Singh, Solid State Commun. 114, 15 (2000).

[29] E. E. Krasovskii, Phys. Rev. B 56, 12866 (1997).

[30] T. Kotani and M. van Schilfgaarde, Solid State Commun. 121, 461 (2002).

[31] A. Svane and E. Antoncik, Phys. Rev. B 35, 4611 (1987).

[32] F. J. Schmitte, in: Numerical Data and Functional Relationships in Science and Technology, Landolt-Börnstein, New Series, edited by O. Madelung (Springer, Berlin, 1993), Group III, Vol. 17F, pp. 146-152.

[33] B. A. Efimova, V. I. Kaidanov, B. X. Moizkes, and I. A. Chernik, Sov. Phys. Solid State 7, 2032 (1966).

[34] R. L. Bernick, L. Kleinman, Solid State Commun. 8, 569 (1970).

[35] K. L. I. Kobayashi, Y. Kato, Y. Katayama, and K. F. Komatsubara, Phys. Rev. Lett. 37, 772 (1976).

[36] L. Esaki and P. J. Stiles, Phys. Rev. Lett. 16, 1108 (1966).

[37] B. M. Wojek, R. Buczko, S. Safaei, P. Dziawa, B. J. Kowalski, M. H. Berntsen, T. Balasubramanian, M. Leandersson, A. Szczerbakow, P. Kacman, T. Story, and O. Tjernberg, Phys. Rev. B 87, 115106 (2013). 
[38] G. Henkelman, A. Arnaldsson, and H. Jónsson, Comput. Mater. Sci. 36, 254 (2006).

[39] W. A. Harrison, J. Vac. Sci. Technol. 19, 1492 (1979).

[40] V. L. Deringer and R. Dronskowski, Chem. Phys. Chem. 14, 3108 (2013).

[41] K. M. Rabe and J. D. Joannopoulos, Phys. Rev. B 36, 6631 (1987).

[42] S. V. Eremeev, Yu. M. Koroteev, E. V. Chulkov, JETP Lett. 91, 594 (2010).

[43] S. V. Eremeev, G. Bihlmayer, M. Vergniory, Y. M. Koroteev, T. V. Menshchikova, J. Henk, A. Ernst, and E. V. Chulkov, Phys. Rev. B 83, 205129 (2011).

[44] H. Lin, R. S. Markiewicz, L. A. Wray, L. Fu, M. Z. Hasan, and A. Bansil, Phys. Rev. Lett. 105, 036404 (2010).

[45] T. Sato, K. Segawa, H. Guo, K. Sugawara, S. Souma, T. Takahashi, and Y. Ando, Phys. Rev. Lett. 105, 136802 (2010).

[46] K. Kuroda, M. Ye, A. Kimura, S. V. Eremeev, E. E. Krasovskii, E. V. Chulkov, Y. Ueda, K. Miyamoto, T. Okuda, K. Shimada,
H. Namatame, and M. Taniguchi, Phys. Rev. Lett 105, 146801 (2010).

[47] Y. L. Chen, Z. K. Liu, J. G. Analytis, J. H. Chu, H. J. Zhang, B. H. Yan, S. K. Mo, R. G. Moore, D. H. Lu, I. R. Fisher, S. C. Zhang, Z. Hussain, and Z. X. Shen, Phys. Rev. Lett. 105, 266401 (2010).

[48] K. Kuroda, M. Ye, E. F. Schwier, M. Nurmamat, K. Shirai, M. Nakatake, S. Ueda, K. Miyamoto, T. Okuda, H. Namatame, M. Taniguchi, Y. Ueda, and A. Kimura, Phys. Rev. B 88, 245308 (2013).

[49] S. V. Eremeev, Yu. M. Koroteev, and E. V. Chulkov, JETP Lett. 91, 387 (2010).

[50] S. V. Eremeev G. Landolt, T. V. Menshchikova, B. Slomski, Y. M. Koroteev, Z. S. Aliev, M. B. Babanly, J. Henk, A. Ernst, L. Patthey, A. Eich, A. A. Khajetoorians, J. Hagemeister, O. Pietzsch, J. Wiebe, R. Wiesendanger, P. M. Echenique, S. S. Tsirkin, I. R. Amiraslanov, J. H. Dil, and E. V. Chulkov, Nat. Commun. 3, 635 (2012). 